https://doi.org/10.23913/ride.v10i19.503

Artículos Científicos

\title{
Innovación y transferencia de tecnología en México. Un análisis empírico de datos panel
}

\author{
Scientific Innovation And Transfer Of Technology In Mexico. A factor \\ analysis of data panel
}

\begin{abstract}
Inovação e transferência de tecnologia no México. Uma análise empírica do painel de dados
\end{abstract}

\author{
Omar Alejandro Pérez Cruz \\ Universidad de Colima, México \\ omar_perez@ucol.mx \\ https://orcid.org/0000-0003-3367-8259
}

\section{Resumen}

La innovación y la transferencia tecnológica en México no siempre se generan en el ámbito empresarial, pues en su génesis intervienen diversos agentes sociales que solo de forma habitual trabajan de manera coordinada, lo que arroja débiles resultados. Por ello, el objetivo de este texto fue analizar el papel que juega la transferencia de conocimientos desde las universidades en la determinación de la innovación tecnológica en México. Metodológicamente, los datos se diseñaron tipo panel, analizados mediante mínimos cuadrados con errores estándar corregidos (MCO), y la base de datos utilizada corresponde al Instituto Mexicano para la Competitividad (IMCO). En tal sentido, los hallazgos indican una relación significativa y positiva entre la innovación y la actividad de las grandes empresas, los posgrados de calidad y los centros de investigación; así como una relación significativa y negativa con las pequeñas y medianas empresas. Por ello, se concluye que 


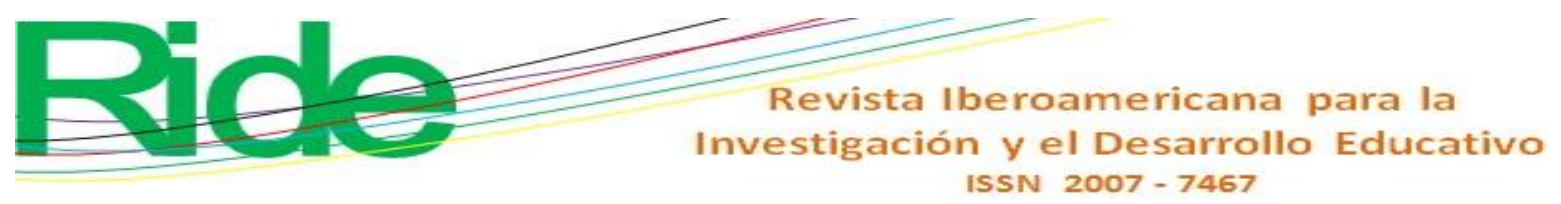

estas características se traducen en importantes áreas de oportunidad en materia de política educativa, su vinculación con grandes empresas y la transferencia del conocimiento generado en universidades del país.

Palabras clave: datos panel, innovación científica, propiedad intelectual, transferencia tecnológica.

\section{Abstract}

Innovation and technology transfer in Mexico are not always generated in the business world. In its genesis, various social agents intervene that do not always work in a coordinated manner, obtaining weak results. That is why, the objective of this research is to analyze the role played by the transfer of knowledge from universities in the determination of technological innovation in Mexico. Methodologically, the data were designed as a panel, analyzed using least squares with corrected standard errors (OLS). The database used corresponds to the Mexican Institute for Competitiveness (IMCO). The results indicate a significant and positive relationship between innovation and the activity of large companies, quality postgraduate programs and research centers. As well as a significant and negative relationship with small and medium enterprises. These characteristics translate into important areas of opportunity in terms of educational policy, its linkage with large companies and the transfer of knowledge generated in universities in the country.

Keywords: panel data, scientific innovation, intellectual property, technology transfer.

\section{Resumo}

A inovação e a transferência de tecnologia no México nem sempre são geradas no ambiente de negócios, pois em sua gênese intervêm diversos agentes sociais que trabalham apenas de maneira coordenada, o que produz resultados fracos. Portanto, o objetivo deste texto foi analisar o papel da transferência de conhecimento das universidades na determinação da inovação tecnológica no México. Metodologicamente, os dados foram elaborados como um painel, analisados por meio de mínimos quadrados com erros padrão corrigidos (MCO), e o 


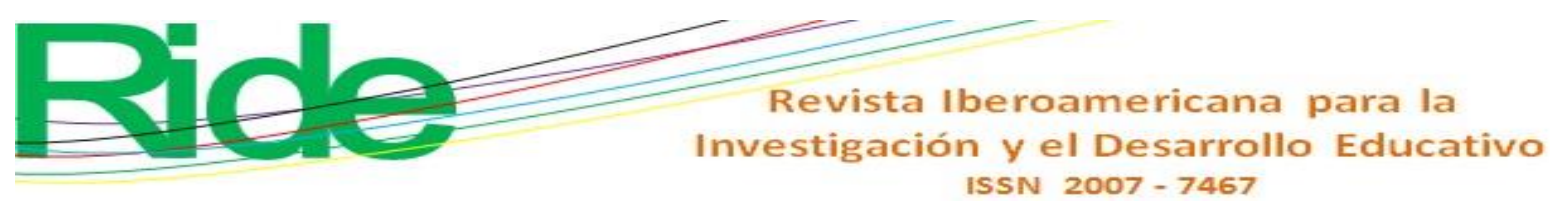

banco de dados utilizado corresponde ao Instituto Mexicano de Competitividade (IMCO). Nesse sentido, os achados indicam uma relação significativa e positiva entre a inovação e a atividade de grandes empresas, cursos de pós-graduação de qualidade e centros de pesquisa; bem como uma relação significativa e negativa com as pequenas e médias empresas. Portanto, conclui-se que essas características se traduzem em importantes áreas de oportunidade no campo da política educacional, sua relação com as grandes empresas e a transferência de conhecimento gerado nas universidades do país.

Palavras-chave: painel de dados, inovação científica, propriedade intelectual, transferência de tecnologia.

Fecha Recepción: Febrero 2019

Fecha Aceptación: Agosto 2019

\section{Introducción}

El fortalecimiento de la relación universidad-empresas es una estrategia con la que algunos países han enfrentado los retos derivados de la innovación que demanda la globalización económica. Con esto buscan incrementar la eficiencia de las empresas e impulsar la competitividad de los sectores económicos. Lógicamente, esta vinculación entre las universidades y los sectores productivos no es reciente, ya que, desde hace décadas, se han implementado fórmulas orientadas a fortalecer este vínculo, aunque los resultados no han sido alentadores, sea por falta de flexibilidad o carencia de voluntad de los agentes para coordinarse.

La transferencia de tecnología ha sido promovida desde el ámbito público, lo que ha servido en México para implementar políticas nacionales y locales que, en términos generales, no han ofrecido los resultados esperados. Entre estas políticas se encuentran la creación del Instituto Nacional del Emprendedor (Inadem, 2019), el Fondo a la Innovación Tecnológica del Consejo Nacional de Ciencia y Tecnología (Conacyt, 2019a), la Conferencia Nacional de Ciencia, Tecnología e Innovación (CNCTI, 2019) o los distintos programas de apoyos a la innovación empresarial (Conacyt, 2019b). Todas estas estrategias son parte de una iniciativa vertical que procura el desarrollo de la capacidad instalada para incrementar la

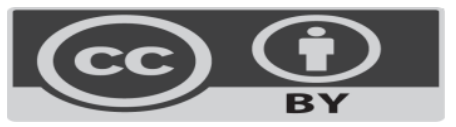




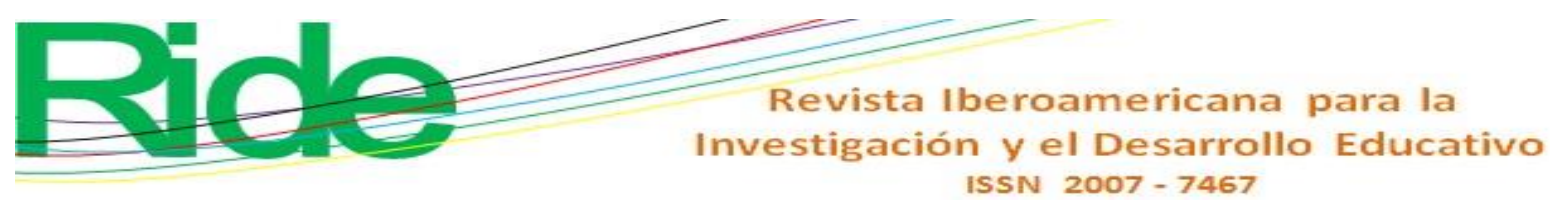

innovación de acuerdo con la American Energy \& Manufacturing Competitiveness Partnership (AEMC, 2013). La hipótesis sugiere que la innovación genera mejoras en la productividad, lo cual produce un incremento en los salarios y, por ende, en la calidad de vida de los trabajadores. Sin embargo, estas políticas no logran integrar a todos los agentes de la triple hélice (por lo menos de manera colaborativa), por lo que cada uno de estos se orienta al logro de resultados de manera aislada, con lo cual se consiguen impactos mínimos y se diluyen los esfuerzos científicos y productivos del país.

Por este motivo, en el presente trabajo se analizó el papel que juega la transferencia de conocimientos desde las universidades en la determinación de la innovación tecnológica en México. Los actores involucrados en este proceso son el gobierno, el sector educativo y el sector productivo. De este modo, las variables se agruparon en dependientes (patentes, como tangible de la innovación) e independientes (empresas, grandes empresas, posgrados de calidad, centros de investigación e investigadores). El periodo de análisis abarcó desde el año 2005 hasta el año 2017 —n este último se encontró la información más actual ofrecida por el Instituto Mexicano para la Competitividad (IMCO)—.

\section{Los principales actores en el proceso de vinculación}

\section{Gobierno}

Desde principios del siglo XXI, la administración pública ha cambiado de una visión neopública a una neogerencial. Esta última concepción posweberiana trasciende el control presupuestal y se enfoca en le eficiencia integral, la transparencia, el acceso a la información y la incorporación de la ciudadanía en el quehacer gubernamental. En este sentido, Cabello y Ortiz (2013) comentan que este cambio de paradigma puede encuadrarse en tres áreas: 1) el nivel de injerencia que el Estado ha tenido en el mercado, 2) la influencia de la tecnocracia sobre la política pública y 3) la separación entre teoría y práctica en el ejercicio de la gestión pública.

Fonseca-Retana, Lafuente-Chryssopoulos y Mora-Esquivel (2016) explican que las políticas públicas continúan con la visión de un modelo lineal para la innovación, pues centran la estrategia en incrementar la oferta educativa. Sin embargo, a pesar del incentivo 


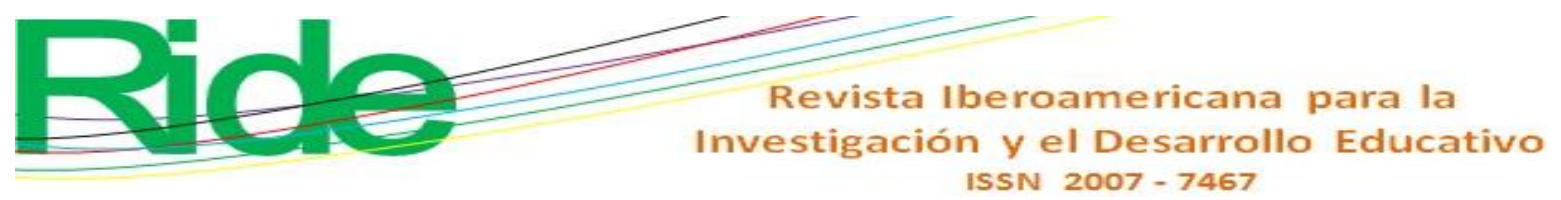

por la oferta de conocimientos, las condiciones de innovación en la investigación y el desarrollo (I+D) no son halagüeñas. Al respecto, Cabrero, Cárdenas, Arellano y Ramírez (2011) señalan que el sistema de desarrollo de innovaciones en México presenta graves privaciones en lo referente al capital humano especializado, carencia de recursos financieros en apoyo a las actividades de I+D, así como una ineficiencia en la productividad investigativa de las instituciones de educación superior.

De acuerdo con Licona y Pérez (2018), para que un país destaque en el escenario internacional, debe centrar su estrategia de desarrollo en el fortalecimiento de un capital humano altamente especializado, porque esto le permite elevar su fuerza laboral y la formación de personal especializado en determinadas áreas estratégicas para la innovación y el desarrollo tecnológico. En resumen, la política de innovación enfocada en ampliar la oferta educativa solo desgasta a la economía y contribuye a la ineficiencia del proyecto económico nacional.

Por ello, el papel del Estado debe cambiar de una postura regulatoria a una de facilitador para convertirse en el puente que enlace el proceso de transferencia de tecnología con el sector productivo. Así generaría un ecosistema de innovación mediante la orientación de políticas verticales en áreas estratégicas de la ciencia y la tecnología.

\section{Sector productivo}

$\mathrm{El}$ actual panorama comercial exige a las empresas mejorar sus procesos e innovar con nuevos productos y servicios. La diferenciación, como estrategia competitiva, demanda una gran cantidad de recursos que pueden resultar costosos para una empresa. Esto significa que para impulsar una innovación de forma individual se debe pensar en los diversos riesgos, como pérdida de competitividad y altos costos de investigación y desarrollo. Por tal motivo, las empresas deben identificar nichos de mercado en los que puedan participar con costos bajos, para lo cual, sin embargo, deben conocer el segmento, pues de ese modo identificarán oportunidades de desarrollo con productos o servicios innovadores.

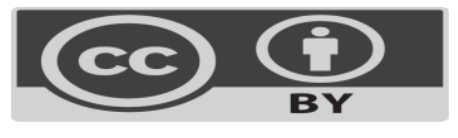



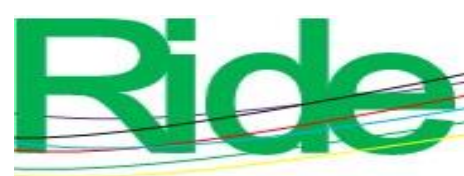

Revista Iberoamericana para la Investigación y el Desarrollo Educativo ISSN $2007-7467$

En otras palabras, para los negocios actuales es importante conocer el contexto donde se desenvuelven para realizar una planeación estratégica que les permita diferenciarse en productos y servicios y apalancar su posicionamiento en el mercado. De lo contrario, la empresa se estaría limitando a imitar a otras compañías líderes en el sector, lo cual podría opacar su posición en el mercado.

En este sentido, Rautu, Racoviteanu y Dinet (2017) explican que algunas empresas que buscan elevar su competitividad solo se enfocan en reproducir lo que hacen las demás, lo cual no constituye benchmarking y tampoco contribuyen a su sobrevivencia en el mercado. Por eso, Welsh, Tullar y Nemati (2016) señalan que un factor clave del éxito empresarial se halla en la formación del empresario, pues de esta forma él podrá ser consciente de cómo puede impulsar la innovación tecnológica de su compañía para enfrentar los retos y los cambios del entorno económico.

\section{Sector educativo}

La dinámica comercial y la velocidad con que se generan las trasformaciones en la tecnología y en determinados campos del quehacer científico han motivado cambios en las concepciones sobre el quehacer educativo y el papel que juegan las universidades en el nuevo escenario mundial. Actualmente, las universidades e instituciones de educación superior deben permanecer como sistemas abiertos, dinámicos y dispuestos al cambio. De hecho, la vinculación entre las universidades y el sector empresarial podrá aumentar los niveles de desarrollo económico y social de un estado, pues esto permitirá crear nuevas líneas de generación y aplicación del conocimiento, así como políticas de evaluación sobre la pertinencia de la investigación, lo que fomentará una educación de calidad y más ajustada a las necesidades de las empresas de la región.

En este sentido, De Arteche, Santucci y Welsh (2013) explican que la vinculación comienza a partir de liderazgos que promuevan nexos entre las universidades y los centros de investigación con las empresas y los sectores productivos de la región. Esta idea se sustenta en lo expuesto por Sharifi Liu y Ismail (2013), quienes concluyen que el establecimiento de estrategias exitosas de vinculación depende en gran medida de los 
gerentes de las oficinas de transferencia del conocimiento en las universidades, cuya visión puede ser vital para la transformación. En otras palabras, el fortalecimiento de esta vinculación y la eficacia de las estrategias de transferencia dependen del capital relacional, es decir, de cómo los actores de las instituciones y los sectores empresariales se asocian eficazmente.

Para ello, las universidades deben apoyar su responsabilidad social en el trabajo que realizan las oficinas de transferencia y fortalecerlas con el nivel adecuado en la estructura organizacional de la institución. Asimismo, estructurar procedimientos y reglamentaciones internas en materia de vinculación con las empresas, así como especificar la manera en que los investigadores generan conocimiento. Además, fomentar políticas de propiedad intelectual y estrategias de emprendimiento e innovación tecnológica.

En este orden de ideas, es importante destacar que el capital humano es un elemento clave en las relaciones universidad-empresa. De hecho, al contar con investigadores altamente especializados que desarrollen innovaciones con apoyo de estudiantes se garantiza una educación de calidad, pertinente y centrada en las necesidades del entorno, ya que el conocimiento y el capital humano son los factores más importantes para integrar cualquier estrategia de innovación y transferencia.

En resumen, en el contexto cambiante de la mundialización, las universidades deben canalizar el conocimiento generado y direccionarlo a los sectores empresariales. De este modo, la academia como agente social y económico pasa de tener un rol pasivo - enfocado en la generación de conocimientos- a asumir un rol activo en la dinámica económica de la región, con lo cual adquiere una posición estratégica en el ámbito económico y político.

\section{Materiales y métodos}

La estructura básica de los modelos de datos panel (MDP) busca estimar si las variaciones observadas en $y$ se relacionan con las variaciones observadas en $x$, tomando en cuenta las diferencias individuales:

$$
\gamma_{i t}=\chi_{i t}^{\prime} \beta+\varepsilon_{i t} \text { ecuación (1) }
$$



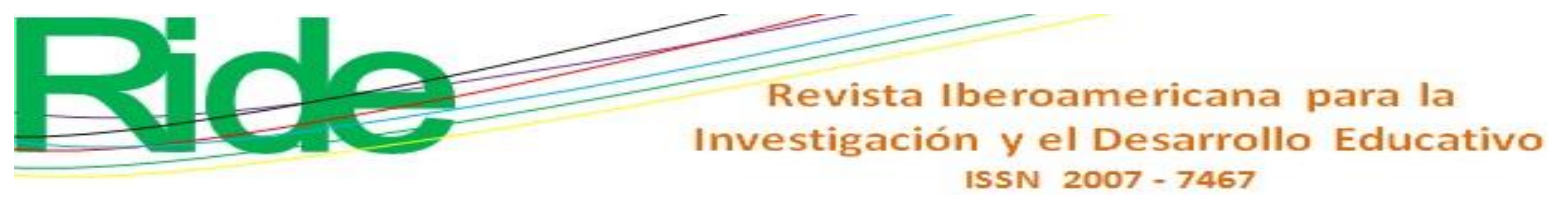

donde $Y$ es el vector de parámetros, $X_{i t}$ es un vector de $k$ variables explicativas; $i: 1$, $\ldots, n$ denota las unidades muestrales, $t: 1, \ldots, T$ indica los periodos, $k: 1, \ldots, \beta$ representa las covariables, $\varepsilon_{i t}$ los errores aleatorios.

De esta manera, se emplearon datos anuales sobre las patentes, empresas, grandes empresas, posgrados de calidad, centros de investigación e investigadores, durante el periodo 2014-2017, para estimar un MDP conformado por 378 municipios de México, especificado por medio de la ecuación (2):

$$
\gamma_{i t}=\mathrm{d}_{i}+\beta_{1} E_{i t}+\beta_{2} F_{i t}+\beta_{3} G_{i t}+\beta_{4} H_{i t}+\beta_{5} J_{i t}+\varepsilon_{i} \quad \text { ecuación (2) }
$$

donde el subíndice $(i)$ refiere los municipios del panel y el subíndice $(t)$ se refiere al periodo de tiempo. El parámetro (d) recoge los componentes determinativos, como el intercepto, lo que permite efectos fijos entre municipios y tendencias. La innovación está representada por $(y), \beta$ representa las covariables, que en este caso son $(E)$ para las empresas, $(F)$ para las grandes empresas, $(G)$ para los posgrados de calidad, $(H)$ para los centros de investigación y $(J)$ para los investigadores. Finalmente, $(e)$ refiere al error aleatorio, con media cero, varianza constante y no autocorrelacionado.

Así, la figura 1 muestra que el diseño de estimación toma como variable dependiente las patentes como elementos de la innovación, mientras que las variables independientes son las covariables antes mencionadas. 
Figura 1. Variables independientes y dependientes

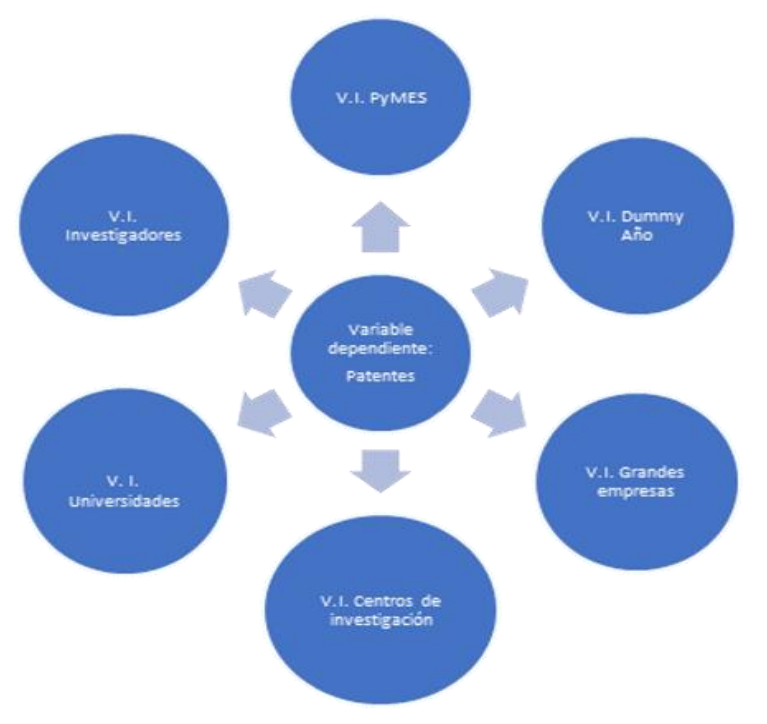

Fuente: Elaboración propia

Específicamente, en la tabla 1 se definen las variables utilizadas en función de los factores económicos y educativos.

Tabla 1. Definición de variables

\begin{tabular}{|l|l|}
\hline \multicolumn{1}{|c|}{ Variables } & \multicolumn{1}{c|}{ Definiciones } \\
\hline Patentes & Número de patentes solicitadas \\
\hline Pymes & Número de registros patronales en el IMSS \\
\hline Grandes empresas & Número de empresas que están del top 500 CNN \\
\hline Posgrados de calidad & Número programas de posgrado certificados por Conacyt \\
\hline Centros de investigación & Número de centros (clave SCIAN 5417) \\
\hline Investigadores & Miembros del SIN \\
\hline
\end{tabular}

Fuentes: IMCO (2018)

Los datos empleados corresponden al índice de competitividad urbana 2018 desarrollado por el Instituto Mexicano de Competitividad (IMCO). Asimismo, las variables se especifican en logaritmos naturales con el fin de corregir problemas de escala y varianza. 

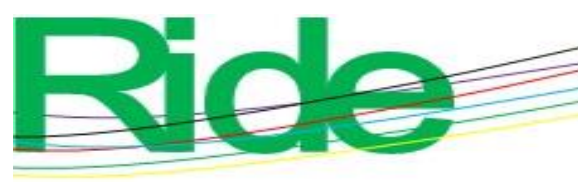

Revista Iberoamericana para la

Investigación y el Desarrollo Educativo ISSN $2007-7467$

La metodología utilizada en esta investigación corresponde a la regresión agrupada: mínimos cuadrados ordinarios (OLS, por sus siglas en inglés). Este es un enfoque simple para analizar datos tipo panel, que omite las dimensiones del espacio y del tiempo de los datos agrupados. Sobre este punto, Cox, Popken y Berman (2013) señalan que en las ecuaciones de regresión es común que se presente el problema de asociaciones espurias en datos panel. Para eso, es necesario determinar el orden de integración y el balance de las variables que conforman el modelo para conocer si las variables están balanceadas o no.

En concordancia con esto, se aplicó al modelo presentado la prueba de Wooldridge y de Wald, la cual permite determinar la existencia de autocorrelación y heterocedasticidad para detectar y controlar posibles problemas de este tipo. La aplicación de las diferentes pruebas señaladas permitió minimizar la heterocedasticidad y la autocorrelación no observable del panel de datos. De este modo, el estimador que se aplicó en esta investigación fue el de errores estándar corregidos para datos panel (EEECP) (Baltagi y Maasoumi, 2018).

\section{Resultados}

En primer lugar, se realizó una primera corrida de las variables para determinar si eran significativas con la variable dependiente. Así, la aplicación de las diferentes etapas de la metodología de OLS permitió corroborar las heterogeneidades no observables del panel de datos, tratando de controlar el carácter individual de cada entidad y corregir, si era el caso, los problemas de heterocedasticidad y autocorrelación que puede presentar el modelo.

En este orden de ideas, en la búsqueda por establecer el mejor estimador para datos tipo panel a partir de las pruebas anteriores, se encontraron los resultados que se muestran en seguida. Antes, vale acotar que este tipo de estimador se ha utilizado en varias investigaciones con datos tipo panel como en series de tiempo. Al respecto, Kachlami y Yazdanfar (2016) y Yao, Chen, Che, Wang y Guan (2018) demostraron que este estimador es más preciso para los datos panel, tal como se muestra en la tabla 2. 
Tabla 2. Innovación y transferencia de conocimientos en México

\begin{tabular}{|l|r|r|}
\hline Variable dependiente & & \\
\hline Patentes & & \\
\hline Variables independientes & Coeficiente & Std. Err. \\
\hline Empresas & $-.0141^{* *}$ & .0072 \\
\hline Grandes empresas & $.1590^{*}$ & .0392 \\
\hline Posgrados de calidad & $.7146^{* *}$ & .3337 \\
\hline Centros de investigación & $26.97^{* *}$ & 13.4884 \\
\hline Investigadores & -.0114 & \\
\hline $\begin{array}{l}\text { Número de observaciones } \\
\text { R-cuadrada }\end{array}$ & 756 & \\
\hline
\end{tabular}

* Significativa $1 \%$. ** Significativa $5 \%$. *** Significativa $10 \%$.

Nota: En los anexos se puede verificar la captura de pantalla del software STATA 14.

Fuente: Elaboración propia con base en STATA 14

\section{Discusión}

Los resultados muestran que las variables más significativas con orientación positiva a la variable dependiente fueron estas: centros de investigación, posgrados de calidad y grandes empresas. En cambio, empresas fue la variable significativa con orientación negativa. La variable investigadores no tuvo relación significativa con la variable dependiente. En la figura 2 se aprecian estas relaciones de manera más clara. 


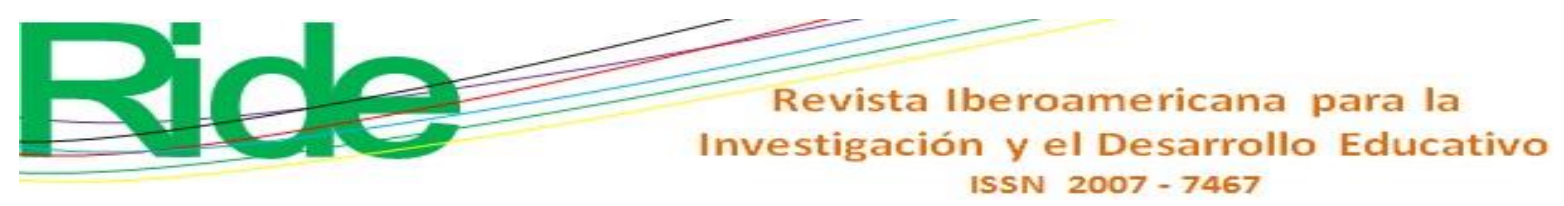

Figura 2. Gráfica de tendencias

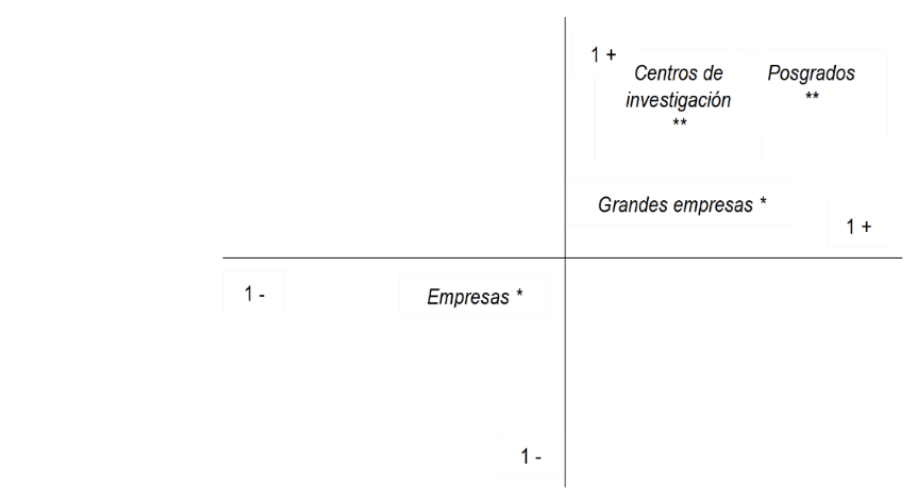

* Significativa $18 \%$. ** Significativa $5 \%$. *** Significativa $10 \%$.

Fuente: Elaboración propia

En relación con la orientación del signo negativo, se puede argumentar que se explica una relación inversa, es decir, a menor presencia de la variable empresa mayor posibilidad de innovación. Estos resultados se refuerzan con los postulados de Olazaran, Albizu, Lavia y Otero (2013), quienes estudiaron la innovación en las pequeñas y medianas empresas (pymes) de la comunidad de Navarra, España. En ese estudio encontraron que si bien la formación y la preparación del personal tienen una aportación sobresaliente, los procesos de apoyo a la innovación se encuentran muy limitados. De hecho, aunque estas empresas tienen un rol incipiente en los procesos de innovación, el sistema de innovación y transferencia de conocimientos, en general, se encuentra desarticulado entre estas y los centros de investigación, así como las instituciones de educación superior. Estas últimas afirmaciones de la desvinculación confirman también los datos arrojados en la presente investigación, pues se observó que no resultaron significativas las variables entre centros de investigación, universidades, patentes y empresas.

Siguiendo con las pymes, Gligo (2016) señala que estas empresas presentan graves problemas, como menor productividad relativa, bajos salarios, falta de personal calificado, escaso nivel de exportaciones y poco acceso a financiamiento. Todos estos factores inherentes a este tipo de empresas obstaculizan la innovación empresarial, disminuyen la inversión en actividades de innovación y desarticulan la vinculación con universidades y centros de investigación. De hecho, los resultados significativos y con orientación positiva 


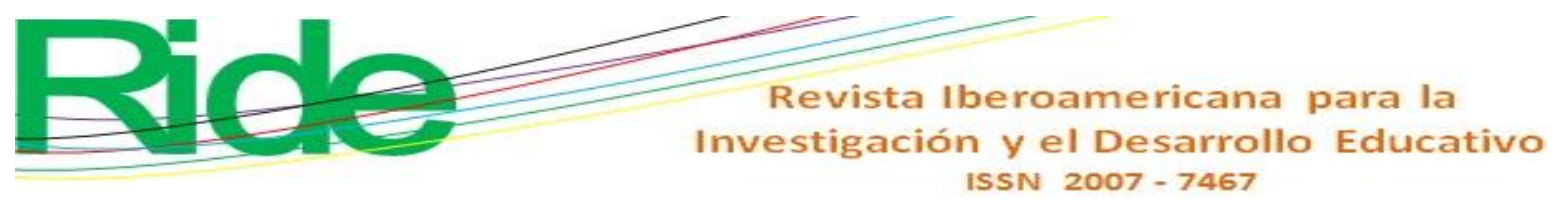

refuerzan lo expuesto anteriormente por Gligo (2016) y Olazaran et al. (2013), ya que el clúster integrado por grandes empresas, universidades y centros de investigación es la triada de innovación y transferencia de tecnología.

Por ello, las grandes empresas centran sus estrategias, generalmente, en el reforzamiento de sus ventajas competitivas mediante la estrategia de diferenciación, para lo cual invierten en investigación y desarrollo, lo que redunda en la generación de patentes. De este modo, las grandes empresas con alto desarrollo e industrialización en sus procesos se ubican en un lugar privilegiado por encima de sus competidores, elevando así las barreas de entrada para cualquier nueva incursión en su segmento de mercado. De acuerdo con Lemley y Feldman (2016) y Zhang y Gallagher (2016), las grandes empresas constituyen un nuevo poderío económico y financiero, ya que sus actividades de innovación desafían la codificación y la estandarización, lo que les permite ser intensivas en conocimientos, exclusivas en derechos de uso y explotación, así como altamente redituables.

Por otro lado, la globalización les permite posicionar su poderío en diferentes países, al ubicar sus centros de producción en naciones con beneficios fiscales y laborales, con menos gastos y exención de impuestos. Los efectos de esta globalización se dejan ver de manera tanto positiva como negativa, ya que si bien son indudables los avances de los sistemas informáticos, las nuevas tecnologías, así como las biotecnologías, entre otros, también se debe subrayar el bajo índice de bienestar y el deterioro ambiental del suelo y del agua. La innovación y la transferencia, por tanto, no siempre ofrecen un bien común, pues se debe recordar que las innovaciones también han servido para acentuar los efectos negativos en los múltiples conflictos bélicos.

Un concepto importante que subyace en los resultados encontrados en esta investigación es que la fuerte relación entre la triada de la innovación se da por redes de conocimiento, el cual transfiere lo generado en las universidades y los centros de investigación hacia las empresas y los sectores productivos de la región.

En este sentido, para explicar el desarrollo de redes de vinculación, es pertinente retomar el concepto de asimilación expuesto por Dávila (2007). Este autor señala, en el marco de las innovaciones científico-tecnológicas, que la asimilación permite observar la velocidad con que las empresas y los países incorporan estos avances tecnológicos en torno 


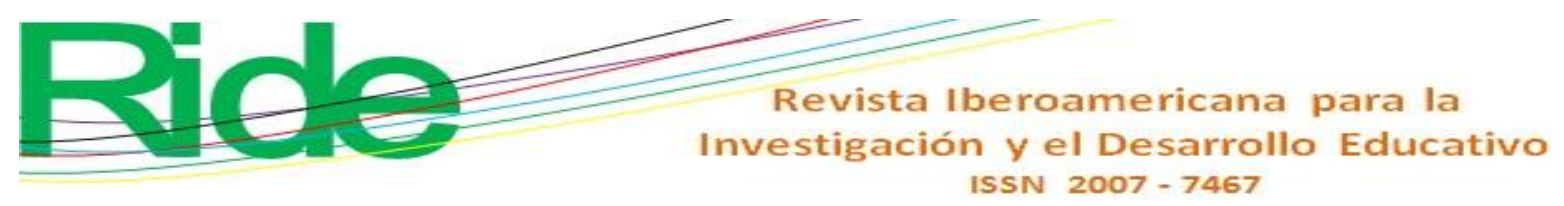

a la ciencia y la tecnología, lo que impulsa la investigación, el desarrollo y, por consiguiente, la innovación.

Sin embargo, no solo es importante incorporar las innovaciones a las operaciones de las empresas, ya que esto solo generaría la adquisición de tecnología, lo que las haría dependientes. Por ello, para la innovación resulta importante la creatividad para resolver necesidades con elementos propios, sin recurrir a la imitación de estrategias, procedimientos o técnicas. De este modo, las innovaciones y las transferencias tecnológicas se dan entre centros de investigación, universidades y grandes empresas, aunque la transferencia se realiza conforme a los requerimientos de estas empresas, por lo que ellas son las propulsoras del conocimiento y ejercen control según sus propios intereses.

En síntesis, los resultados encontrados por la estimación econométrica muestran que los factores estudiados son consistentes con otras investigaciones mencionadas anteriormente y corroboran lo predicho por los modelos de innovación tecnológica, ya que a mayor relación entre universidades, centros de investigación y grandes empresas se tienen efectos positivos y significativos en la innovación tecnológica.

\section{Conclusiones}

El objetivo de esta investigación fue analizar el papel que juega la transferencia de conocimientos desde las universidades en la determinación de la innovación tecnológica en México. Para esto se aplicó un modelo econométrico de MCO que procuró estimar la función de la transferencia de conocimientos; asimismo, se agruparon datos tipo panel (en el periodo 2005-2017) con las variables pymes, grandes empresas, universidades, centros de investigación e investigadores.

De los resultados arrojados en esta investigación, se muestra claramente que los postulados planteados por los modelos de innovación tecnológica se cumplen de manera significativa y positiva en el caso de México. Los resultados econométricos de la función de innovación proporcionan evidencia empírica suficiente del papel relevante de las universidades, los centros de investigación y las grandes empresas en la generación de innovaciones, situación que es postulada en diversas investigaciones referidas.

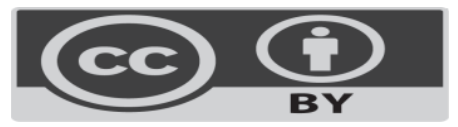


Finalmente, con base en los resultados obtenidos del análisis empírico de la función de las patentes, se verificó que la transferencia de tecnología desde las universidades y los centros de investigación impactan de manera positiva en la innovación del país. Sin embargo, y aun cuando estos efectos resultan significativos, los esfuerzos de transferencia tecnológica todavía son insipientes en México, por lo que esta vía de crecimiento sostenido puede representar un impacto en la economía en el país.

\section{Referencias}

AEMC (2013). Rebuilding the Industrial Commons. Recuperado de de https://www.compete.org/storage/images/uploads/File/PDF\%20Files/AEMC_Prime r_FINAL.PDF.

Baltagi, B. and Maasoumi, E. (2018). An Overview of Dependence in Cross-Section, TimeSeries, and Panel Data. Econometric Reviews, 32(5/6), 543-546. Doi:10.1080/07474938.2012.740957

Cabello, A. y Ortiz, E. (2013). Políticas públicas de innovación tecnológica y desarrollo: teoría y propuesta de educación superior. Convergencia, 20(61), 135-172.

Cabrero, E., Cárdenas, S., Arellano, D. y Ramírez, E. (2011). La vinculación entre la universidad y la industria en México. Una revisión a los hallazgos de la Encuesta Nacional de Vinculación. Perfiles Educativos, 33, 187-199.

CNCTI (2019). Conferencia Nacional de Ciencia, Tecnología e Innovación. Recuperado de https://www.conacyt.gob.mx/index.php/el-conacyt/conferencia-nacional-de-cienciatecnologia-e-innovacion.

Conacyt (2019a). Fondo a la innovación tecnológica. Recuperado de https://www.conacyt.gob.mx/index.php/el-conacyt/convocatorias-y-resultados conacyt/convocatorias-fondos-sectoriales-constituidos/convocatoria-se-conacyt innovacion-tecnologica/convocatorias-cerradas-se-conacyt-innovacion tecnologica/convocatoria-se-conacyt. 

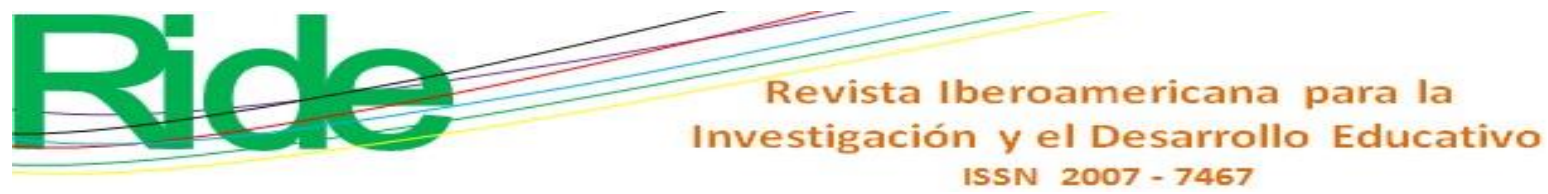

Conacyt (2019b). Apoyos a proyectos de innovación empresarial. Recuperado de https://www.conacyt.gob.mx/index.php/el-conacyt/desarrollo-tecnologico-einnovacion.

Cox, L., Popken, D. and Berman, D. (2013). Causal versus spurious spatial exposureresponse associations in health risk analysis. Critical Reviews in Toxicology, 43, 2638. Doi:doi.org/10.3109/10408444.2013.777689

Dávila, F. (2007). Ciencia, transferencia e innovación tecnológica en Estados Unidos, La Unión Europea y Japón en la era de la globalización. México: Fontamara.

De Arteche, M., Santucci, M. y Welsh, V. (2013). Redes y clusters para la innovación y la transferencia del conocimiento. Impacto en el crecimiento regional en Argentina. Estudios Gerenciales, 29(127), 125-272. Doi:10.1016/j.estger.2013.05.001

Fonseca-Retana, L., Lafuente-Chryssopoulos, R. y Mora-Esquivel, R. (2016). Evolución de los modelos en los procesos de innovación, una revisión de la literatura. Tecnología en Marcha, 29(1), 108-117.

Gligo, N. (2016). Innovación en las pymes de América Latina. Seminario Innovación para pymes exportadoras. Santiago de Chile. Recuperado de https://www.cepal.org/sites/default/files/events/files/nicolo_giglo.pdf.

IMCO (2018). Índice de competitividad urbana. Califica a tu alcalde: manual urbano para ciudadanos exigentes. Recuperado de http://imco.org.mx/indices/califica-a-tualcalde/.

Inadem (2019). Recuperado de https://www.inadem.gob.mx/.

Kachlami, H. and Yazdanfar, D. (2016). Determinants of SME growth: The influence of financing pattern. An empirical study based on Swedish data. Management Research Review, 39(9), 966-986. Doi:10.1108/MRR-04-2015-0093

Lemley, M. and Feldman, R. (2016). Patent Licensing, Technology Transfer, and Innovation. American Economic Review, 106(5), 188-192. Recuperado de https://doi.org/http://www.aeaweb.org/aer/.

Licona, A. y Pérez, O. (2018). El capital humano especializado en la estrategia de ciencia y tecnología en México. Praxis Investigativa ReDIE: Revista Electrónica de la Red 

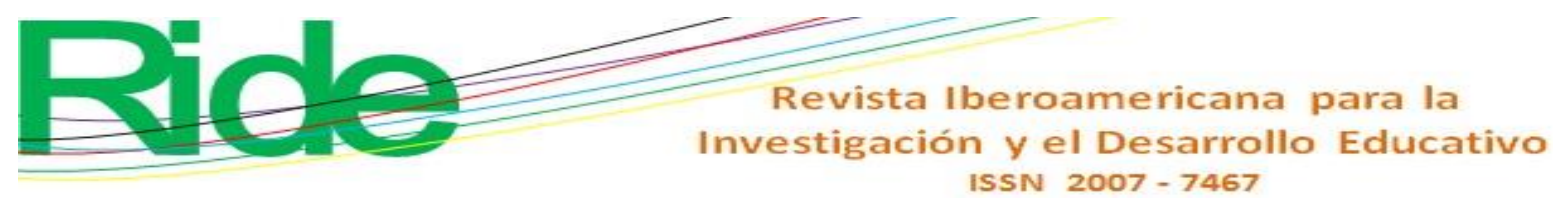

Durango de Investigadores Educativos, 10(19), 8-24. Recuperado de https://dialnet.unirioja.es/servlet/articulo?codigo=6571555.

Olazaran, M., Albizu, E., Lavia, C. y Otero, B. (2013). Formación profesional, pymes e innovación en Navarra. Cuadernos de Gestión, 13(1), 15-40. Doi:10.5295/cdg.110290mo

Rautu, R., Racoviteanu, G. and Dinet, E. (2017). Use of Benchmarking For the Improvement of the Operation of the Drinking Water Supply Systems. Procedia Engineering, 209, 180-187. Doi:10.1016/j.proeng.2017.11.145

Sharifi, H., Liu, W. and Ismail, H. (2013). Higher education system and the 'open' knowledge transfer: a view from perception of senior managers at university knowledge transfer offices. Studies in Higher Education, 39(10), 1860-1884. Doi:10.1080/03075079.2013.818645

Welsh, D., Tullar, W. and Nemati, H. (2016). Entrepreneurship education: Process, method, or both? Journal of Innovation \& Knowledge, 1(3), 125-180. Doi:10.1016/j.jik.2016.01.005

Yao, Y., Chen, D., Che, L., Wang, H. and Guan, Q. (2018). A time series of urban extent in China using DSMP/OLS nighttime light data. PLoS ONE, 13(5), 1-24. Doi:10.1371/journal.pone.0198189

Zhang, F. y Gallagher, K. (2016). Innovation and technology transfer through global value chains: Evidence from China's PV industry. Energy Policy, 94(1), 191-203. Doi:10.1016/j.enpol.2016.04.014 


\section{Revista Iberoamericana para la Investigación y el Desarrollo Educativo ISSN 2007 - 7467}

\section{Anexos}

Captura de pantalla del análisis MCO, STATA 14

Linear regression, heteroskedastic panels corrected standard errors

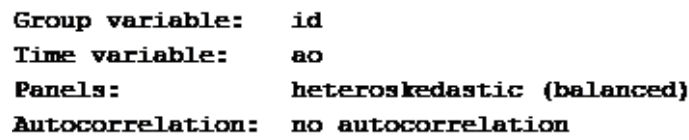

$\begin{array}{lrr}\text { Number of obs } & = & 756 \\ \text { Number of groups } & = & 378 \\ \text { Obs per group: } & \\ \text { min } & = & 2 \\ \text { avg } & = & 2 \\ \max & = & 2 \\ & = & 0.8915 \\ \text { R-squared } & = & 148.14 \\ \text { Dald chi2 (5) } & = & 0.0000 \\ \text { Prob }>\text { chi2 } & & \end{array}$

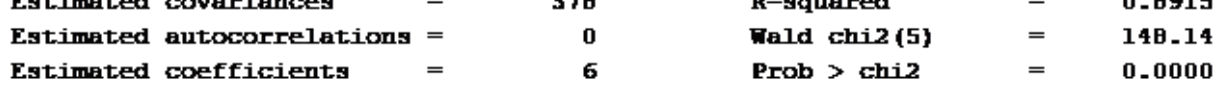

\begin{tabular}{|c|c|c|c|c|c|c|}
\hline \multirow[b]{2}{*}{ patentes } & \multicolumn{3}{|c|}{ Het-corrected } & \multirow[b]{2}{*}{$\mathbf{B} \mathbf{| z |}$} & & \multirow[b]{2}{*}{ Interval] } \\
\hline & Coef - & Std. Err. & $\mathbf{z}$ & & [95? Conf. & \\
\hline empresas & -.0141885 & -0072376 & -1.96 & 0.050 & -.028374 & $-3.10=-06$ \\
\hline grandesempresas & -1590647 & -0392793 & 4.05 & 0.000 & -0820787 & -2360507 \\
\hline posgradosdecalidad & -7146558 & $-333759 \theta$ & 2.14 & 0.032 & -0604987 & 1.368813 \\
\hline Centrosdeinvestigación & 26.97725 & 13.48B4B & 2.00 & 0.045 & -5403177 & 53.41418 \\
\hline investigadores & -.0114595 & -0195993 & -0.58 & 0.559 & -.0498735 & -0269544 \\
\hline cons & -2.437507 & 26.33411 & -0.09 & 0.926 & -54.05141 & 49.17639 \\
\hline
\end{tabular}

